Research Article,

\title{
Retrospective Analysis of COVID 2020 Epidemiological Data in the Town of Kardzhali
}

\author{
Violeta Yordanova1 $^{1}$, Slaveija Dimitrova ${ }^{1}$, Magdalena Marinova ${ }^{1,2}$, Diana Valcheva ${ }^{1}$, Viktor Kirchev ${ }^{2}$ \\ Todor Tcherkezov ${ }^{1,3}$ \\ ${ }^{1}$ MPHAT "Dr. Atanas Dafovski” Kardzhali \\ ${ }^{2}$ Regional Health Inspection- Kardzhali \\ ${ }^{3}$ With affiliation of the Department of Public Health at the Medical University-Sofia, Bulgaria \\ E-mail Address: dribrahimeke@gmail.com
}

\begin{abstract}
:
Objectives: We aim to present the retrospective results and statistical analysis of a year under COVID19 pandemic. Such analyses might be of great importance for management of individual cases of the disease on a day-to-day basis, as well as deciding upon certain restrictive measures as a whole; border-crossings movement, diagnostic testing and limiting travels in order to contain the pandemic of Covid 19. We hope our data contributes for further epidemiological investigations and future reference information.

Design and methods: We combined collected information from our rtPCR test results, statistical data within our hospital and epidemiological data according to our Regional Health Inspection. The collected data is cumulative and statistical analysis incredibly helpful and necessary. Our Molecular Diagnostics Unit is licensed within the territory of Bulgaria. Our methods were strict protocols from the guidelines of National Center for Infectious and Parasitic Diseases-Bulgaria; the kits we use are all CE, the overall concept in sync with the global regulations as from the CDC and the WHO. Statistical analyses were done with ANOVA tool.

Results: We give an overview of the collected data over the months of pandemic for the year 2020, as we observe several increases in the numbers of infected patients, but instead of having graphs with distinctive peaks we demonstrate a steady increase, giving a glimpse of the magnitude of the pandemic that followed after. The rtPCR test results are presented by pool data for both healthy travelling individuals and patients for confirming Covid diagnosis.

Discussion: The current report represents retrospective data and suggests the possibilities, available to nations and applicable locally and of immense importance near border-crossing regions in a situation of pandemic with such magnitude and without the immediate access to vaccines and reliable treatment resources for novel infectious outbreaks.
\end{abstract}

Keywords: COVID19, SARS-CoV-2, global pandemic, border-crossings restrictions.

\section{Introduction:}

\section{Background and summary}

In the beginning of the year 2020 the news of the new and deadly disease broke. We only had limited information of this brand-new virus from the family of the Coronaviruses; some vague specifics we had yet to discover. Swiftly it became evident, the entire world would be affected and will partake in the fight against it, as well as describing the symptoms, the major manifestations, treating patients, testing, preventing and contributing to the global knowledge in the prevention and cure. We established our COVID unit testing lab within the Microbiology and Virology Division in the main County Hospital in the town of Kardzhali, Eastern 
Rodopi, Bulgaria, Eastern Europe. Upon the beginning of our new line of work, we immediately started testing huge amounts of inpatients and outpatients as we did our best to provide prompt service, deliver results the same day, report to physicians and local health authorities for the appropriate measures and prescription of quarantine to whoever tested positive. We have been working according to mandatory regulations for border crossings globally [1] and locally [2,3] and provided standard testing, plus verified result certification. Globally, the migration and border crossings regulations were newly determined and for Europe each and every country had established bulletin and informative data as recommendation of migration given [4]. Most sites were updated if not daily, quite frequently, due to the ever changing environment. Important aspect globally was presented by authors, suggesting increased tension in areas with higher rates of migration with population emerging from Wuhan as the main infection source in other cities and provinces. In China some cities with a low number of cases showed a rapid increase in case load. Owing to the upcoming Spring Festival return wave, understanding the risk trends in different regions is crucial to ensure preparedness at both the individual and organization levels and to prevent new outbreaks [5]. In the USA since the outbreak, the FBI anticipated a rise in hate crimes across the United States citing examples such as the stabbing of an Asian American family, including children ages 2 and 6, whom the assailant believed were spreading COVID-19. Their prediction was confirmed by the Asian Pacific Policy \& Planning Council, with documented over 1,000 reports from Asian people of coronavirus discrimination and hate crimes from March 19th to April 1st of last year. Common incidents included verbal harassment, shunning, and physical assault. These accounts, along with associations between "Chinese" and "virus", suggest the emergence of a more sinister phenomenon-namely, the personification of COVID-19 as Asian people. This is especially tragic for Asian healthcare workers, who make up $17 \%$ of physicians in active practice and are the most represented ethnic group among foreignborn medical professionals currently in the USA [6].
According to the authors in the viral article "The Hummer and the Dance" [6] and the implications - " if the governments could buy us more time", "Strong coronavirus measures today should only last a few weeks, there shouldn't be a big peak of infections afterwards, and it can all be done for a reasonable cost to society, saving millions of lives along the way. If we don't take these measures, tens of millions will be infected, many will die, along with anybody else that requires intensive care, because the healthcare system will have collapsed".

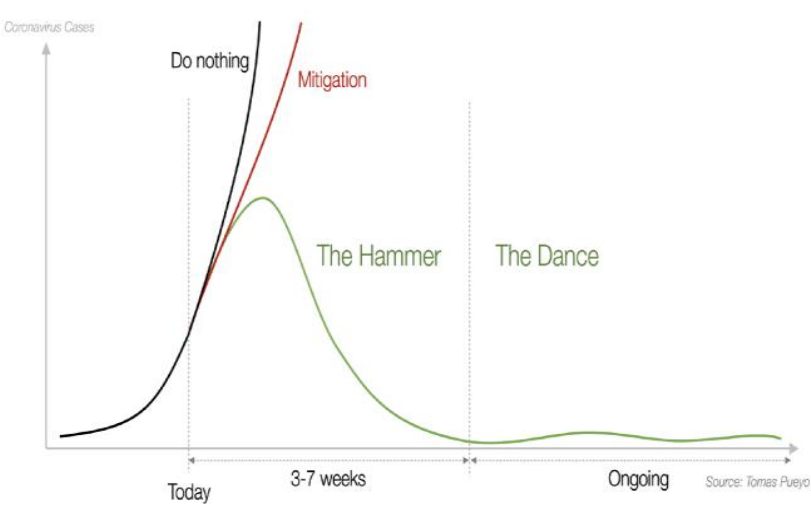

Figure 1. Suppression vs Mitigation vs Do Nothing early on

As the current progress of the SARS-CoV-2 outbreak has made obvious, clinical management of the infection has been under multiple pressures ranging from high false-negative rates of diagnosis, contentious results on the efficacy of proposed drugs, and the threat of severely detrimental symptoms like ARDS and cytokine storm responses. The development of accessible point-of-care diagnostic devices tailor-made for low-resource settings continues to be a pressing requirement. Certain points have been recurrent the advantages of leveraging existing knowledge on closely related species like the SARS CoV, the potent efficacy of seemingly disparate techniques when applied in tandem, and the importance of collaborative efforts coupled with rapid, timely dissemination of results which may inform ongoing efforts. Requirements key to enabling these include the availability of genomic sequences, structural data, and expertly curated databases. The push towards developing modular assay workflows (with elements of automation involved) may well be a defining paradigm of future responses to such scenarios, according to [7]. 
Other authors express definitive opinions regarding Covid-19, as being undoubtedly a public health catastrophe and certainly calls for enhanced investments in $R \& D$ that impinges directly on public health. But more fundamentally Covid-19 pandemic reflects fundamental ecological disequilibrium. Evidence has accumulated that loss of biodiversity and everincreasing human incursions into the natural world has contributed heavily to the outbreak and spread of epidemic diseases. Understanding the three Es-evolution, ecology and the environmentwill be key to identifying potential pandemics. Covid-19 also reinforces the need to pay far greater attention to the bio-sciences that underpin agriculture, health and the environment that are going to be profoundly impacted by the current pandemic [8]. The epidemiological situation in Bulgaria is a part of the global pandemic of the Corona virus disease COVID-19, caused by the SARS-CoV-2 virus. The first isolated case in Bulgaria was from March, 8 of 2020 and the first patient admitted to the hospital for active treatment MPHAT "Dr. Atanas Dafovski" was from March $25^{\text {th }}$, 56 years old male from Kardzhali. Since the beginning of the pandemic, an unified protocol for patients to be admitted in

\section{Methods:}

Table 1 Data, presenting the number of patients admitted/discharged/deceased each month at the hospital in Kardzhali.

\begin{tabular}{|l|l|l|l|l|l|l|}
\hline Months & $\begin{array}{l}\text { Sick } \\
\text { patients }\end{array}$ & Admitted & Discharged & Deceased & $\begin{array}{l}\text { Remained } \\
\text { sick }\end{array}$ & $\begin{array}{l}\text { Realized } \\
\text { bedridden } \\
\text { numbers }\end{array}$ \\
\hline March & 0 & 3 & 0 & 0 & 3 & 8 \\
\hline April & 3 & 4 & 4 & 1 & 2 & 72 \\
\hline May & 2 & 2 & 4 & 0 & 0 & 68 \\
\hline June & 0 & 14 & 8 & 1 & 5 & 117 \\
\hline July & 5 & 26 & 18 & 3 & 10 & 249 \\
\hline August & 10 & 17 & 18 & 1 & 8 & 244 \\
\hline September & 8 & 36 & 28 & 3 & 13 & 328 \\
\hline October & 13 & 92 & 58 & 17 & 30 & 627 \\
\hline November & 30 & 151 & 95 & 45 & 41 & 1182 \\
\hline December. & 41 & 202 & 166 & 25 & 52 & 1748 \\
\hline Total & $\mathbf{0}$ & $\mathbf{5 4 7}$ & $\mathbf{4 0 1}$ & $\mathbf{9 6}$ & $\mathbf{5 2}$ & $\mathbf{4 6 4 3}$ \\
\hline
\end{tabular}

The accumulation of data in our hospital was grouped and thoroughly analyzed. According to it, we have the following numbers, presented in Table 1: hospital was established, based upon the medical anamnesis, rtPCR or $\mathrm{Ag}$ test positive result, airways insufficiency, shortness of breath, febrile, $\mathrm{x}$-Ray of lungs, showing obstructions and specific Covid patterns, CAT scan data to determine the percentage of affected pulmonary parenchyma, laboratory data from blood gas, $\mathrm{O}^{2}$ saturation and overall score of the condition of the patient. After hospitalization and treatment, the exit is either improvement for the patient with regression of the affected by the virus pulmonal tissue, increased $\mathrm{O}^{2}$ saturation, compared to the initial one and to be $>93 \%$, or lethal exit, with irreversible outcome, heavy infection and $>75 \%$ affected pulmonary tissue. Key element in the outcome plays the presence of concomitant diseases, as the greatest burden comes from the diabetes, heart conditions and many more. In practice we see more patients in younger age develop heavier immunological reaction, when exposed to the infectious agent, which is the main reason behind the pathogenesis of the heavy fulminant forms of disease manifestation.

The current paper aims to report retrospective data from tested patients, hospitalized individuals and cumulative information over the months of the past 2020. 
in numbers of the admitted patients, as well as high morbidity and mortality (Fig 2).

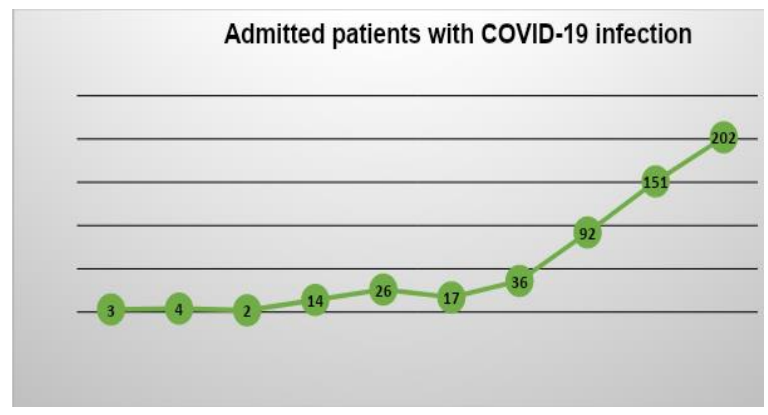

Figure 2 Admitted patients for treatment into the MPHAT "Dr. Atanas Dafovski"; each dot presents data for each month from March to December

Since the beginning of the epidemiological measures in sync with the global pandemic of Coronavirus disease COVID-19 the Hospital for Active Treatment "Dr. Atanas Dafovski " admitted for treatment 547 patients with COVID-19 identified or unidentified virus. The discharged patients to date have realized bedridden numbers totaling 4643 as the overall length of hospitalization for each patient was 8,5 days. Of the discharged patients $399(80 \%)$ were with COVID-19 proven virus, whereas 98 (20\%) unidentified. The ratio man/women was $262 / 230$, in Fig. 3.

Distrubution of discharged patients with COVID-19 male to female

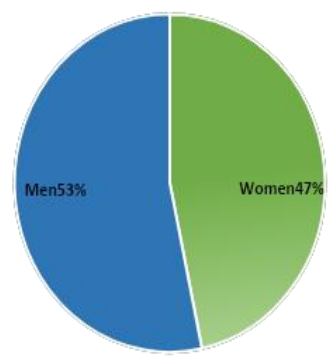

Figure 3 Data for the gender distribution in the patients with COVID19 at the hospital "Dr. Atanas Dafovski"

The vast majority of the treated patients with COVID19 were aged over 65 years, as their percentage was $52 \%$. In the hospital there was only one child, age 15 and the oldest patients were female, age 92 and male, age 91.

The distribution in sex and age is being presented in Table 2:
Table 2 Data for the number distribution of patients for sex and age.

\begin{tabular}{|l|l|l|l|l|l|}
\hline $\begin{array}{l}\text { Sex/ } \\
\text { Age }\end{array}$ & $\begin{array}{l}\text { 18and } \\
\text { under }\end{array}$ & $\begin{array}{l}\text { Up } \\
\text { to 30. }\end{array}$ & $\begin{array}{l}\text { From } \\
30 \text { to 50. }\end{array}$ & $\begin{array}{l}\text { From } \\
50 \text { to 65 }\end{array}$ & $\begin{array}{l}\text { 65 } \\
\text { and up }\end{array}$ \\
\hline Women & 1 & 3 & 25 & 86 & 119 \\
\hline Men & & 1 & 43 & 80 & 139 \\
\hline
\end{tabular}

In our hospital there were patients, treated for COVID-19 that were mainly residing in the region of Kardzhali and the adjacent district. The vast groups of patients admitted were from the Kardzhali region. The biggest portion of the distribution in terms of residence of the patients, the ones from Kardzhali city were greatest-- 212 and the district of Kardzhali - 76, followed by the Kirkovo municipality district -67 and Ardino municipality- 25 .

Summarized data were presented in Table 3:

Table 3 Data for the patients' distribution per residence

\begin{tabular}{|l|l|}
\hline Region/Municipality & Number of patients \\
\hline Kardzhali & 288 \\
Ardino & 25 \\
Dzhebel & 19 \\
Kirkovo & 67 \\
Krumovgrad & 28 \\
Momchilgrad & 21 \\
Chernoochene & 24 \\
Haskovo district & 10 \\
Sofia & 5 \\
Others & 10 \\
\hline
\end{tabular}

The deceased with COVID-19 to November 2020 were 26 and the last couple of months the mortality increased dramatically, as in November alone they were 45 and in December 25. In the ICU after intensive treatment and resuscitation there were 72 dead patients in total. The data is shown in fig. 4. 


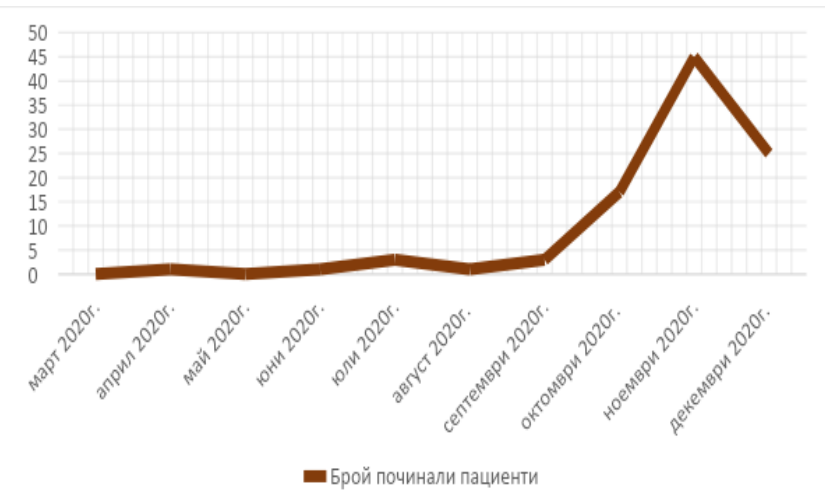

Figure 4 Data for the deceased patients each month for the MPHAT "Dr. Atanas Dafovski"

The collected information and the terrible statistics are on trend with the data, shown for the rest of the territory of Bulgaria, according to which for the last couple of months for the 2020 we have record high numbers of victims of the COVID-19.

The incredibly high number of deaths for any country with a population under 7 million citizens isn't a novelty, given the continuous increase in numbers of new registered cases of COVID19 and this trend continues throughout the end of 2020.

The victims of the virus are only one side of the picture, as we have even more alarming data from the statistics of the National Statistical Institution for the total number of deaths in weeks for the country during the year 2020. They show increase of the death cases after mid October, as in November the number of the deaths for the 2019 were 8115, and for the year 2020-; 16044 . For the month of December the data shows 8340 in 2019 and 15917 deaths for 2020 .

The deaths in our hospital was $8 \%$ during the month of November and $6 \%$ for the month of December in 2020, as with the normal values of $2 \%$ for the last decade.

The mortality data fromCOVID-19 in percentage at our hospital were as follows: November: $24,9 \%$., December - $10,3 \%$ and the total percentage since the beginning of the pandemic were- $17,6 \%$.

The distribution in terms of sex and age for the death patients is presented bellow in fig. 5 and 6:

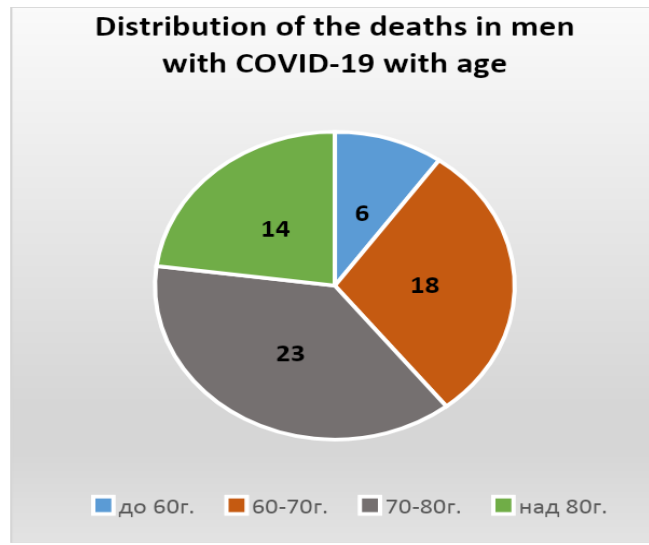

Figure 5 Data for the deceased men

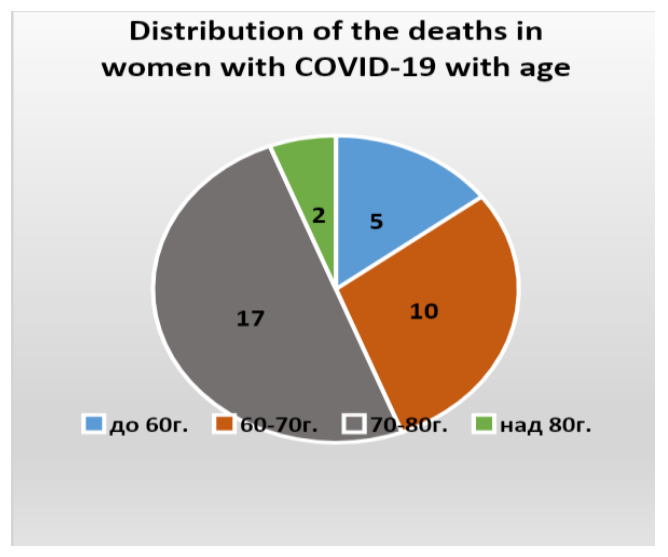

Figure 6 Data for the deceased women

The majority of the deceased were with concomitant chronic diseases, most common of which were diabetes mellitus, as 11 patients were with an oncological preexisting condition.

The distribution of the patients in the documents described illnesses is presented in Table 4:

Table 4 Data of the concomitant diseases of the patients at MPHAT “Dr. Atanas Dafovski”

\begin{tabular}{|l|l|}
\hline Illness & $\begin{array}{l}\text { Numberof } \\
\text { patients }\end{array}$ \\
\hline Diabetes mellitus & 122 \\
Hypertension & 150 \\
Chronic cardiac disease & 69 \\
Chronic pulmonary disease & 35 \\
Cerebrovasculardisease & 18 \\
complications & 11 \\
Malignancies & 6 \\
Chronic kidney disease & \\
\hline
\end{tabular}


At the microbiological laboratory at the hospital the rtPCR testing has been initiated since the month of May for the SARS-CoV2 infection. Since the start of that particular kind of testing till the end of the year 2020 we have done 6956,-
rtPCR tests and fast serum antigen tests -287 and fast antigen tests- 46, of which 2358 for inpatients and 4931 for outpatients.

The data for the tested patients is presented in Fig.

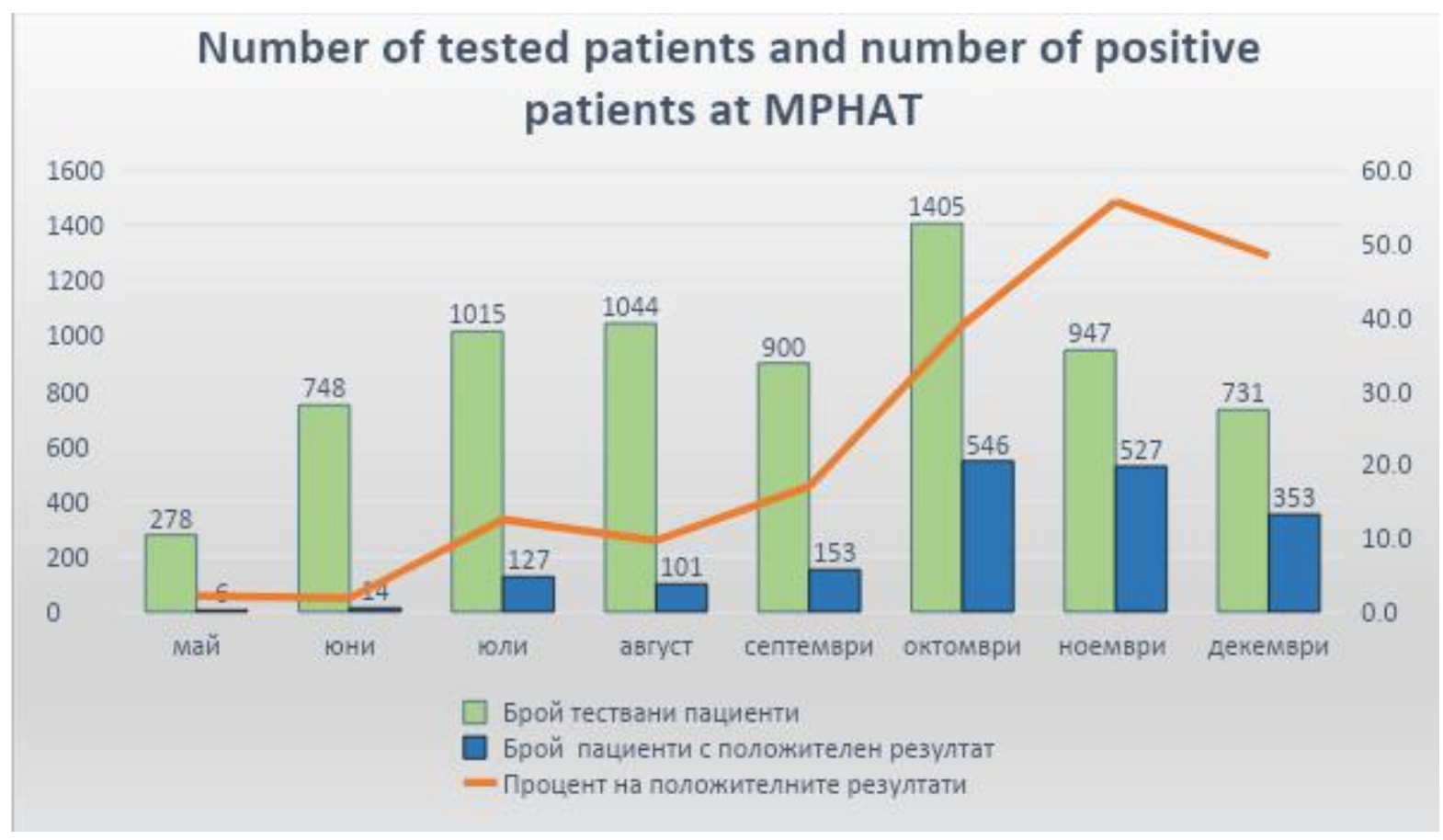

Figure 7 Data for the tested patients for SARS-CoV2 infection each month (data taken from annual hospital report). Bar sets give monthly statistics from May to the left, and December to the very right at the graph. Green bars represent total number tested each month, blue bars are the positive and the orange line gives percentage of positive results.

Data for the medical staff at MPHAT "Dr. Atanas Dafovski” admitted and treated for COVID-19 infection (according to the data from the centralized register of COV.ID)

1. Doctors -10 .

2. Nurses -13 .

3. Physicians' assistants -1 .

4. Other medical personnel -4 .

5. Patients care aids -3 .

6. Staff aid at day care- 1 .

\section{Discussion:}

Since our data has been collected daily and reported to local and national authorities, we continue to study the trends and nuances of the mass number of tested individuals. Although we separate and count the foreign passports in our database, we firmly believe the major reason for testing is for travel across borders. We cannot tell though what percentage of the bulgarian passportholder healthy individuals, subjected to testing, had the intention of traveling and therefore we can only speculate, based on the data we currently have, the trend shows the evidence of spread due to people's travel and movement. Based on our evidence, we can make a suggestion that we observe a higher percentage of infected, tested positive and ultimately sick patients in the larger cities near border crossings, where the testing for SARS-Cov2 is being done.

As we experienced several complete lockdowns so far, followed by slight removal of restrictions we 
can only hope we follow the scheme outlined by "The Hammer and the Dance". Our greatest hope with providing quality medical care and services and with more medicinal products now available to specifically target the COVID infection, more and more people would take the advantage and to be slowly moving back to normalcy.

\section{Conclusion:}

The retrospective analysis of the data represents theexact evidence and gives a valuable overview of past situations. In the current state of unknown we look at the data as an important tool in predicting the possible scenarios of further developments and this information gives us the preparedness to face challenges and overcome difficult times when battling with the disease.

We dedicate our research to all the health care workers, the doctors, nurses, patient care personnel, laboratory technicians, receptionists, security services and to express gratitude for their compassionate work and highly professional approach in this pandemic.

\section{References:}

[1] Observatory on Border Crossings Status due to COVID-19 Homehttps://wiki.unece.org/display/CTR BSBC/Bulgaria\#page-banner starthttps://bnr.bg/en/post/101307437/co vid-19-at-bulgaria-greece-border-arenot-mandatory

[2] New Greece-Bulgaria border checkpoint makes for safer, faster travel https://ec.europa.eu/regional_policy/en/p rojects/Greece/new-greece-bulgariaborder-checkpoint-makes-for-saferfaster-travel

[3] Julián Alfredo Fernández-Niño 1, Andrés Cubillos-Novella 2, IetzaBojórquez 3, Michael Rodríguez 4Recommendations for the response against COVID-19 in migratory contexts under a closed border: The case of Colombia Affiliations expand PMID: 33152189 PMCID: PMC7676824 DOI: 10.7705/biomedica.5512

[4] H Alexander Chen 1, Jessica Trinh 1, George P Yang 23 Anti-Asian sentiment in the United States - COVID-19 and history PMID: 32425201 PMCID:
PMC7229717

DOI: 10.1016/j.amjsurg.2020.05.020

[5] Ze-Liang Chen 12, Qi Zhang 3, Yi Lu 4, Zhong-Min Guo 5, Xi Zhang 3, Wen-Jun Zhang 6, Cheng Guo 7, Cong-Hui Liao 1, Qian-Lin Li 1, Xiao-Hu Han 2, JiaHai Lu 1Distribution of the COVID-19 epidemic and correlation with population emigration from Wuhan, China PMID: 32118644 PMCID: PMC7147281 DOI: 10.1097/CM9.0000000000000782

[6] Coronavirus: The Hammer and the Dance What the Next 18 Months Can Look Like, if Leaders Buy Us Time

[7] Review COVID-19: Advances in diagnostic tools, treatment strategies, and vaccine development $M$ SREEPADMANABH, AMIT KUMAR SAHU and AJIT CHANDE* Molecular Virology Laboratory, Indian Institute of Science Education and Research, Bhopal, India *Corresponding author (Email, ajitg@iiserb.ac.in) MS received 26 June 2020; accepted 15 October 2020

[8] Opinion Science \& technology in a postCovid-19 world JAIRAM RAMESH MP and Chairman of Parliament's Standing Committee on Science \& Technology, Environment, Forests \& Climate Change, New Delhi, India (Email, jairam54@gmail.com) 